\title{
c-MET receptor-targeted fluorescence on the road to image-guided surgery in penile squamous cell carcinoma patients
}

Hielke M. de Vries ${ }^{1,2}$, Elise Bekers ${ }^{3}$, Matthias N. van Oosterom ${ }^{1,2}$, M. Baris Karakullukcu4 ${ }^{4}$, Henk G. van der Poel $^{1}$, Fijs W.B. van Leeuwen ${ }^{1,2}$, Tessa Buckle ${ }^{1,2^{*}}$, Oscar R. Brouwer ${ }^{1,2^{*}}$

\footnotetext{
${ }^{1}$ Department of Urology, Netherlands Cancer Institute, Amsterdam, the Netherlands

${ }^{2}$ Interventional Molecular Imaging Laboratory, Department of Radiology, Leiden University Medical Centre, Leiden, the Netherlands

${ }^{3}$ Department of Pathology, Netherlands Cancer Institute, Amsterdam, the Netherlands

${ }^{4}$ Department of Head and Neck Surgery, Netherlands Cancer Institute, Amsterdam, the Netherlands

* These authors contributed equally to the establishment of this manuscript.
}

\section{Corresponding Authors:}

Oscar R. Brouwer, MD, PhD, FEBU

Plesmanlaan 121, 1066 CX Amsterdam, The Netherlands

o.brouwer@nki.nl

Tessa Buckle, PhD

Albinusdreef 2, 2333ZA Leiden, The Netherlands

$\underline{\text { T.Buckle@lumc.nl }}$

\section{First Author:}

Hielke M. de Vries, MD, PhD student

Plesmanlaan 121, 1066 CX Amsterdam, The Netherlands

hm.d.vries@nki.nl

Word count abstract: 300

Word count text: 4695 
Funding: This research was financially supported by a Netherlands Organization for Scientific Research TTW-VICI grant (Grant No. TTW 16141). EMI-137 was provided by Edinburgh Molecular Imaging (EMI), KARL STORZ provided the Cy5 fluorescence camera, and the FIS-00 near-infrared fluorescence camera was made available by Hamamatsu photonics.

Short running title: c-MET fluorescence penile cancer imaging 


\section{ABSTRACT}

In penile squamous cell carcinoma ( $\mathrm{pSCC}$ ), primary surgery aims to obtain oncologically safe margins while minimizing mutilation. Surgical guidance provided by receptor-specific tracers could potentially improve margin detection and reduce unnecessary excision of healthy tissue. Here, we present the first results of a prospective feasibility study for real-time intraoperative visualization of pSCC using a fluorescent mesenchymal-epithelial transition factor (c-MET) receptor targeting tracer (EMI-137).

\section{Methods}

EMI-137 tracer performance was initially assessed ex vivo $(\mathrm{N}=10)$ via incubation of freshly excised pSCC in a solution containing EMI-137 (500 nM). The in vivo potential of c-MET targeting and intraoperative tumour visualization was assessed after intravenous administration of EMI-137 in five pSCC patients scheduled for surgical resection using a Cyanine-5 (Cy5) fluorescence camera. Fluorescence imaging results were related to standard pathological tumour evaluation and c-MET immunohistochemistry. Three of the five in vivo patients also underwent a sentinel node resection after local administration of the hybrid tracer indocyanine green (ICG)- ${ }^{99 m}$ Tcnanocolloid, which could be imaged using a near-infrared fluorescence camera.

\section{Results}

No tracer-related adverse events were encountered. Both ex vivo and in vivo, EMI-137 enabled cMET based tumour visualization in all patients. Histopathological analyses showed that all pSCC's expressed c-MET, with expression levels of $\geq 70 \%$ in $14 / 15$ patients. Moreover, the highest c-MET 
expression levels were seen on the outside rim of the tumours, and a visual correlation was found between c-MET expression and fluorescence signal intensity. No complications were encountered when combining primary tumour targeting with lymphatic mapping. As such, simultaneous use of Cy5 and ICG in the same patient proved to be feasible.

\section{Conclusion}

Fluorescence imaging of c-MET receptor-expressing pSCC tumours after intravenous injection of EMI-137 was shown to be feasible and can be combined with fluorescence-based lymphatic mapping. This combination is unique and paves the way towards further development of this surgical guidance approach.

\section{Keywords}

Penile squamous cell carcinoma, fluorescence-guided surgery, c-MET receptor, receptor-targeted imaging, sentinel node, multicolour 


\section{INTRODUCTION}

Surgical resection remains the mainstay of treatment for primary penile squamous cell carcinoma (pSCC). The potential morbidity of surgery, and the impact on the patient's sexuality and quality of life, can be minimized by performing penile-sparing surgery in early disease (1). Unfortunately, in today's practice, positive margins - potentially causing local cancer recurrence - still occur in up to $36 \%$ of patients (2). Hence, penile-sparing surgery needs to strike an optimal balance between acquiring oncologically safe resection margins and maximizing residual function and appearance.

Penile cancer surgery is one of the common indications wherein image-guided sentinel node resections are applied as routine care (3). The current state of the art herein relies on lymphatic migration of the near-infrared tracer indocyanine green (ICG)-99mTc-nannocolloid after local administration (Figure 1, in green; (3)).

Expression of the c-MET oncogene has been recorded in up to $87 \%$ of pSCC patients (4). Previously, it was shown that use of the far-red (Cyanine-5 (Cy5)) fluorescent mesenchymalepithelial transition factor (c-MET) targeting peptide EMI-137 (formally known as GE-137), in combination with experimental cameras, was able to facilitate receptor-mediated fluorescenceguided resection of c-MET positive lesions during colonoscopy $(0.13 \mathrm{mg} / \mathrm{kg})$ and esophagoscopy (0.09-0.13 mg/kg) (5-7) after intravenous tracer administration (Figure 1, in red).

Recent efforts have shown that commercial fluorescence cameras can be modified slightly to allow for Cy5 imaging and can even be used for multicolour imaging applications that allow visualization of two complementary tracers in the same patient $(8,9)$. 
In this feasibility study in pSCC patients, we evaluated if ex vivo incubation could predict the in vivo success of c-MET receptor-targeted fluorescence-guided imaging and if this technology could be applied simultaneously with fluorescence-guided lymphatic mapping (Figure 1).

\section{MATERIALS AND METHODS}

\section{Patients}

Fifteen patients with clinical suspicion of pSCC were prospectively included for either ex vivo $(\mathrm{N}=10)$ or in vivo $(\mathrm{N}=5)$ evaluation. Patient and tumour characteristics are presented in Table 1. For in vivo assessment of EMI-137 (Edinburgh Molecular Imaging), patients with severe kidney insufficiency and patients who actively planned pregnancy of their partners or abstained from using two forms of barrier contraceptives were excluded. Three of the five in vivo patients also underwent sentinel node resection for expected T1G2 or higher disease with clinically unsuspicious lymph nodes. Pathological TNM stage, primary tumour surgery type, differentiation grade, lymphovascular invasion, perineural growth, the presence of penile intraepithelial neoplasia, radicality, margin size, tumour diameter, infiltration depth, and p16 status were recorded (Table 1). The institutional review board has approved the ex vivo study, and the medical ethical committee approved the in vivo study. All subjects signed an informed consent form. The in vivo study was registered in the European Clinical Trials Register (2019-003022-24). 


\section{Tracer Preparation}

For ex vivo sample assessment, EMI-137 was dissolved in PBS to a concentration of $500 \mathrm{nM}$ (20 $\mathrm{mL}$ per sample). For in vivo use, EMI-137 was prepared for intravenous administration under good manufacturing practices by our institutions' pharmacy. To this end, the content of a vial of EMI137 was reconstituted with $5.0 \mathrm{~mL}$ sterile water, after which the vial contains an isotonic solution of $4.8 \mathrm{mg} / \mathrm{ml} \mathrm{EMI-137} \mathrm{in} \mathrm{a} 50 \mathrm{~m}$ phosphate buffer. On the morning of surgery, the pharmacy department prepared a syringe with the exact EMI-137 dose based on patient weight. Additional detail on the fluorescent tracer EMI-137 tracer and an in-depth description of its pharmacokinetics were previously described by Burggraaf et al. (5).

ICG-99mTc-nanocolloid was prepared according to previously described methods $(3,10)$.

\section{Fluorescence Imaging Modalities}

White light and far-red fluorescence imaging were conducted using a clinical-grade Cy5 fluorescence camera (Karl Storz Endoskope GmbH; (11)) complemented with in-house developed image-processing software that allowed colour-coding of the fluorescence signal for improved visualization and distinction of intensity differences, hereby allowing direct correlation to the tumour-to-background ratio (TBR; the ratio between relative fluorescence units (12) in the tumour and surrounding tissue). This image-processing software was built to run in real-time and was written in C++-programming language. To map differences in fluorescence intensity measured to different colour values, open-source computer vision libraries (OpenCV) were used. The colourmap allowed real-time visualization of the fluorescence signal's distribution within the 
tissue sample (pseudo-coloured fluorescence overlay visible on a separate screen) and representation of the TBR via an intensity-based scalebar (fluorescence signal intensity differences represented via a colour spectrum). Confirmation of the TBR values was obtained using Image J software by dividing the fluorescent signal intensity in the tumour by the fluorescent signal intensity in background tissue.

Near-infrared fluorescence imaging was performed using a FIS-00 fluorescence camera (Hamamatsu photonics).

\section{Ex Vivo Assessment Feasibility c-MET Targeting}

Resected primary tumour specimens were collected directly post-excision and assessed immediately (Figure 2A). Tumour samples were bisected at the pathology department prior to ex vivo imaging. For ex vivo assessment of in vivo targeting feasibility, samples were incubated in a solution containing EMI-137 (500 nM, 20mL) for five minutes. Hereafter, samples were rinsed twice with PBS to clear unbound EMI-137. Samples obtained from patients who received EMI-137 via intravenous administration were imaged without further pre-treatment.

\section{In Vivo Tracer Administration and Fluorescence-guided Imaging}

Five pSCC patients received intravenous administration of EMI-137 $(0.13 \mathrm{mg} / \mathrm{kg} ;(5))$ at 3 hours ( \pm 30 minutes) before surgery. Adverse events were registered up to two weeks after intravenous tracer injection. Three patients also underwent a sentinel node resection based on the local 
intradermal administration of ICG-99m Tc-nanocolloid in three depots and preoperative nodal identification (lymphoscintigraphy and single-photon emission computed tomography with computed tomography (SPECT/CT) imaging at 15 minutes and 2 hours post tracer administration $((3,10)$; Figure 1).

To maintain the standard surgical procedure until the ability of EMI-137 to visualize tumour tissue reliably was demonstrated, the operating surgeon was blinded from the intraoperative far-red fluorescence imaging results. The surgical assistant performed Cy5-based imaging. White light and fluorescence images of the tumour were made immediately before incision at the operation room as well as intra- and post-operatively. Sentinel nodes were resected based on their anatomical location at SPECT/CT, intraoperative radio guidance (with gamma probe), and near-infrared fluorescence guidance. Excised samples were also imaged ex vivo.

\section{Pathological Assessment}

After ex vivo imaging, samples were formalin-fixed and paraffin-embedded. Three $\mu \mathrm{m}$ sections were subjected to either c-MET or hematoxylin and eosin staining. Immunohistochemistry (IHC) of the formalin-fixed paraffin-embedded tumour samples was performed on a BenchMark Ultra autostainer (Ventana Medical Systems). Briefly, paraffin sections were cut at $3 \mu \mathrm{m}$, heated at $75^{\circ} \mathrm{C}$ for 28 minutes, and deparaffinized in the instrument with EZ prep solution (Ventana Medical Systems). Heat-induced antigen retrieval was carried out using Cell Conditioning 1 (CC1, Ventana Medical Systems) for 64 minutes at 950C. c-MET was detected using clone SP44 (Ready-to-Use, 
16 minutes at 360C, Roche Diagnostics). Bound c-MET was detected using the UltraView Universal DAB Detection Kit (Ventana Medical Systems). Slides were counterstained with Hematoxylin and Bluing Reagent (Ventana Medical Systems).

A dedicated urological cancer pathologist scored stained sections. Percentages of positive cells were scored for four staining intensity categories $(-,+,++,+++)$. From this, a c-MET score was calculated by multiplying the percentage of positive tumour area by the intensity of the staining $(1(+) * \%$ positive $+2(++) * \%$ positive $+3(+++) * \%$ positive) $(13)$. In short, a c-MET score is an average expression intensity of the complete tumour area. The possible range of the c-MET score ranges from 0 (no expression at all) to 3 (100\%+++ expression)(13).

Excised lymph nodes were pathologically examined for the presence of macro/micrometastases or isolated tumour cells, according to previously described protocols (3). The resected penectomy specimens were examined and classified for pathologic tumour stage and grade (14).

\section{Statistical Analysis}

For continuous variables, the range is reported. Mann-Whitney $U$ was used to compare TBR between ex vivo and in vivo samples. Spearman correlation was used for comparison of continuous variables. A p-value of $<0.05$ was deemed significant. 


\section{RESULTS}

Initial ex vivo assessment of the feasibility of tumour visualization based on c-MET targeting (Figure 2) resulted in tumour identification in all ten tumour specimens. TBR were in the range of $2.0 \pm 0.6-3.4 \pm 0.3$ (Table 1). The identification of tumour tissue was improved by the real-time conversion of the fluorescence-output to a heatmap-based colour-coding (Figure 2B second from the left and centre). IHC confirmed c-MET expression in all tumours and showed the highest cMET expression at the edge of the tumour (Figure 2B second from the right). Membranous C-MET expression was seen in $70-100 \%$ of cells per specimen (median 90\%), providing c-MET scores in the range of $0.7-2.3$ (median 1.2) (13). Standard pathological processing was not affected by the presence of Cy5 fluorescence in the surgical sample.

Based on the successful targeting of c-MET in ex vivo surgical specimens, EMI-137 was intravenously administered to five patients with pSCC (Figure 3). Far-red fluorescence imaging allowed the identification of the primary tumour (Figure 3B; encircled in black). Again, colourcoding obtained after (real-time) image processing provided increased discrimination between the tumour and the surrounding healthy tissue. No interference of renally cleared EMI-137 was observed (Figure 3B). TBR values after intravenous tracer administration were similar to those reported for the ex vivo incubation, namely between $2.0 \pm 0.4-4.2 \pm 0.9$ (Table 1; p-value = 0.68).

In the three patients who also underwent sentinel node biopsy, preoperatively identified sentinel nodes (Figure 4A-C) were removed using a combination of radio- and fluorescence guidance. Fluorescence imaging prior to resection of the sentinel node enabled visualization of both lymphatic drainage from the injection site (Figure 4D) and superficially located sentinel 
nodes (Figure 4D, encircled in white). The signal intensity increased when the incision was placed, and overlaying tissue was resected. All 11 resected sentinel nodes were tumour-negative at pathology. This was underlined by the presence of a clear ICG-related signal in the sentinel node, while no clear tumour-related Cy5 signal could be detected (Figure 4E). Moreover, while the injection site for ICG-99m Tc-nanocolloid and the location of the tumour were located within the same field of view (Figure 1), near-infrared fluorescence-guidance was shown not to be impaired by the presence of far-red fluorescence, or vice versa (Figure 3B and 4D/E).

Histopathologically, membranous c-MET expression was seen in all tumours (range 30$100 \%$; median $95 \%$ ), with an expression of $\geq 70 \%$ of cells per excised specimen in $4 / 5$ tumours, resulting in c-MET scores in the range of $0.4-2.3$ (median 1.7; Table 1). Comparative to the ex vivo specimens, c-MET expression was present throughout the tumour, but the expression was increased at the tumour edge (Figure 3C).

A (non-significant) trend of rising TBR with an increasing c-MET score was observed (Spearman's correlation coefficient $=0.203, \mathrm{p}$-value $=0.469)$. Interestingly, the c-MET score and TBR appeared to be higher in patients with LVI, T-stage $\geq 2, \mathrm{cN}+$, and $\mathrm{p} 16$ positive patients (Table 1), which may support the hypothesis that especially the patients with a worse tumour stage or human papillomavirus induced tumours might benefit from c-MET-targeted surgery. 


\section{DISCUSSION}

In this pilot study in penile cancer patients, we found that EMI-137, a c-MET specific fluorescent tracer, enabled receptor-mediated ex vivo and in vivo fluorescence-based visualization of primary $\operatorname{pSCC}(\mathrm{TBR}>2$; Table 1$)$. In line with previous literature, no adverse events were recorded after intravenous administration of EMI-137 (5). Furthermore, Cy5-based far-red imaging proved to be compatible with near-infrared fluorescence imaging (ICG-99mTc-nanocolloid) performed during the same procedure.

The finding that all pSCC tumours (over)expressed c-MET $\geq 70 \%$ with preselection of patients and that the tumour tissue could be stained using EMI-137 suggests that c-MET is a potential target for image guidance for pSCC. By blinding the surgeon from the far-red fluorescence imaging result during surgery, the standard surgical procedure remained unaffected, and no decisions that could alter patient treatment were made prior to the assessment of the feasibility of the c-MET targeting approach. However, this approach also unabled the possibility of intraoperative margin assessment. Nevertheless, postoperative assessment underlined the presence of c-MET expression in all samples and that the c-MET-related fluorescence signal was visible at the edge of all tumours (Figures 2 and 3), indicating the possible future potential in this cancer type. The plurality of available fluorescent tracers that are currently evaluated in clinical studies for a variety of tumour types (e.g. pancreatic cancer, head and neck cancer and melanoma) also paves the way for the assessment of other possible imaging targets and targeted tracers for penile cancer (15-21). 
Our study confirms the high c-MET expression rate of $87 \%$ in pSCC found by Gunia et al. (4). In all 15 patients, far-red fluorescence could be related to the presence of the c-MET receptor at IHC. These results are in line with c-MET specific fluorescent tracer reports from studies in Barrett's oesophagus and dysplastic colorectal polyps (5-7). This correlation and high expression pattern make the c-MET receptor potentially a promising target for tumour-specific applications in pSCC. Further investigation in a larger patient cohort will be required to substantiate the correlation between C-MET score and TBR, to investigate the ability to reduce positive surgical margins and to identify the patient group that will benefit most from c-MET based fluorescenceguided surgery.

This study also shows that primary tumour imaging using far-red (Cy5) fluorescence ( $\lambda_{\max }$ ex $=640 \mathrm{~nm} ; \lambda_{\max }$ em $\left.=680 \mathrm{~nm}\right)$ using a first-in-human laparoscopic camera from Karl Storz Endoskope could be performed alongside near-infrared (ICG) imaging of ICG- ${ }^{99 \mathrm{~m}} \mathrm{Tc}$-nanocolloid $\left(\lambda_{\max e x}=800 \mathrm{~nm} ; \lambda_{\max e m}=820 \mathrm{~nm}\right)$ using a clinical-grade fluorescence camera. Fortunate for the patients, the evaluated lymph nodes in this study did not contain metastases, and no Cy5-related signal could be detected in these nodes. While the utility of targeted tracers for visualization of micrometastases can be debated (22), tumour-targeted far-red imaging has been shown to allow SN mapping successfully (20). The combined use of two different fluorescent entities within the same procedure supports the future exploration of multicolour fluorescence-guided surgery avenues (9) that allow visualization of distinct anatomical features (e.g., tumour and sentinel nodes) alongside each other. 


\section{CONCLUSION}

The results from this pilot study suggest that c-MET is a promising target for fluorescence-guided tumour identification, with the potential to improve margin assessment in pSCC. The c-MET targeted approach proved to be compatible with lymphatic mapping performed in the same patient using multicolour fluorescence guidance based on both far-red (Cy5) and near-infrared (ICG) imaging.

\section{Disclosure}

This research was financially supported by a Netherlands Organization for Scientific Research TTW-VICl grant (Grant No. TTW 16141). EMI-137 was provided by Edinburgh Molecular Imaging (EMI), Storz endoscopes provided the Cy5 fluorescence camera, and the FIS-00 near-infrared fluorescence camera was made available by Hamamatsu photonics. The study's funder did not influence data collection, analysis, interpretation, or writing of the manuscript. No other potential conflicts of interest relevant to this article exist.

\section{ACKNOWLEDGMENTS}

We would like to thank Alwin Huitema, Erik van Muilekom, Jeroen Visser, Eva Offringa, Florian van Beurden, Nina Heimburger, Danny van Willigen, Fabian van Hensbergen, Krijn Houwing, and Leon Slof for assistance with the set-up, logistics, conduction, chemistry, and software of this 
study. We also acknowledge the NKI-AVL Core Facility Molecular Pathology \& Biobanking (CFMPB) for lab support.

\section{KEY POINTS}

\section{Question}

Is the c-MET specific tracer EMI-137 feasible for intraoperative visualization of penile squamous cell carcinoma?

\section{Pertinent finding}

This observational fluorescent tracer study describes findings on ten fresh ex vivo tumour specimens and five patients who were intravenously injected with a c-MET specific fluorescent tracer. All tumours were visible via fluorescence imaging, and all expressed c-MET on immunohistochemistry. Thus, c-MET fluorescent imaging appears to be feasible in penile squamous cell carcinoma. Moreover, this c-MET targeted approach proved to be compatible with lymphatic mapping performed in the same patient.

\section{Implications for patient care}

This study indicates that during future pSCC surgery, fluorescent primary tumour visualization is feasible, even in combination with sentinel node procedures. With targeted tracers like EMI-137 and multicolour imaging capabilities, technical capabilities of providing image guidance towards the primary tumour are now emerging. 


\section{REFERENCES}

1. Kieffer JM, Djajadiningrat RS, van Muilekom EAM, Graafland NM, Horenblas S, Aaronson NK. Quality of life for patients treated for penile cancer. J Urol. 2014;192:1105-10.

2. Baumgarten A, Chipollini J, Yan S, et al. Penile sparing surgery for penile cancer: A multicenter international retrospective cohort. J Urol. 2018;199:1233-1237.

3. Dell'Oglio P, de Vries HM, Mazzone E, et al. Hybrid indocyanine Green-99mTcnanocolloid for single-photon emission computed tomography and combined radio- and fluorescence-guided sentinel node biopsy in penile cancer: Results of 740 inguinal basins assessed at a single institution. Eur Urol. 2020;78:865-872.

4. Gunia S, Erbersdobler A, Hakenberg OW, Koch S, May M. C-MET is expressed in the majority of penile squamous cell carcinomas and correlates with polysomy-7 but is not associated with MET oncogene amplification, pertinent histopathologic parameters, or with cancer-specific survival. Pathol Res Pract. 2013;209:215-20.

5. Burggraaf J, Kamerling IMC, Gordon PB, et al. Detection of colorectal polyps in humans using an intravenously administered fluorescent peptide targeted against c-Met. Nat Med. 2015;21:955-61.

6. de Jongh SJ, Voskuil FJ, Schmidt I, et al. C-Met targeted fluorescence molecular endoscopy in Barrett's esophagus patients and identification of outcome parameters for phase-I studies. Theranostics. 2020;10:5357-5367.

7. de Jongh SJ, Vrouwe JPM, Voskuil FJ, et al. The optimal imaging window for dysplastic colorectal polyp detection using c-Met-targeted fluorescence molecular endoscopy. J 
Nucl Med. 2020;61:1435-1441.

8. Schottelius M, Wurzer A, Wissmiller K, et al. Synthesis and preclinical characterization of the PSMA-targeted hybrid tracer PSMA-I\&F for nuclear and fluorescence imaging of prostate cancer. J Nucl Med. 2019;60:71-78.

9. van Beurden F, van Willigen DM, Vojnovic B, et al. Multi-wavelength fluorescence in image-guided surgery, clinical feasibility and future perspectives. Mol Imaging. 2020;19:1-18.

10. Brouwer OR, Buckle T, Vermeeren L, et al. Comparing the hybrid fluorescent-radioactive tracer indocyanine green-99mTc-nanocolloid with 99mTc-nanocolloid for sentinel node identification: a validation study using lymphoscintigraphy and SPECT/CT. J Nucl Med. 2012;53:1034-40.

11. van Willigen DM, van den Berg NS, Buckle T, et al. Multispectral fluorescence guided surgery; A feasibility study in a phantom using a clinical-grade laparoscopic camera system. Am J Nucl Med Mol Imaging. 2017;7:138-147.

12. Tummers WS, Warram JM, van den Berg NS, et al. Recommendations for reporting on emerging optical imaging agents to promote clinical approval. Theranostics. 2018;8:53365347.

13. Mazières J, Brugger W, Cappuzzo F, et al. Evaluation of EGFR protein expression by immunohistochemistry using $\mathrm{H}$-score and the magnification rule: re-analysis of the SATURN study. Lung Cancer. 2013;82:231-7. 
14. O.W. Hakenberg, E. Comperat, S. Minhas, A. Necchi, C. Protzel NW. EAU Guidelines Penile Cancer. 2020.

15. van Leeuwen FWB, Schottelius M, Brouwer OR, et al. Trending: Radioactive and fluorescent bimodal/hybrid tracers as multiplexing solutions for surgical guidance. J Nucl Med. 2020;61:13-19.

16. Hernot S, van Manen L, Debie P, Mieog JSD, Vahrmeijer AL. Latest developments in molecular tracers for fluorescence image-guided cancer surgery. Lancet Oncol. 2019;20:e354-e367.

17. Joshi BP, Wang TD. Targeted optical imaging agents in cancer: Focus on clinical applications. Contrast Media Mol Imaging. 2018;2018:1-19.

18. van Keulen S, Nishio N, Fakurnejad S, et al. Intraoperative Tumor Assessment Using RealTime Molecular Imaging in Head and Neck Cancer Patients. J Am Coll Surg. 2019;229:560567.e1.

19. Tummers WS, Miller SE, Teraphongphom NT, et al. Intraoperative Pancreatic Cancer Detection using Tumor-Specific Multimodality Molecular Imaging. Ann Surg Oncol. 2018;25:1880-1888.

20. Zanoni DK, Stambuk HE, Madajewski B, et al. Use of Ultrasmall Core-Shell Fluorescent Silica Nanoparticles for Image-Guided Sentinel Lymph Node Biopsy in Head and Neck Melanoma. JAMA Netw Open. 2021;4:e211936.

21. Nishio N, van den Berg NS, van Keulen S, et al. Optical molecular imaging can differentiate 
metastatic from benign lymph nodes in head and neck cancer. Nat Commun.

2019;10:5044.

22. van Leeuwen FWB, Winter A, van Der Poel HG, et al. Technologies for image-guided surgery for managing lymphatic metastases in prostate cancer. Nat Rev Urol. 2019;16:159-171. 


\section{Figure legends}

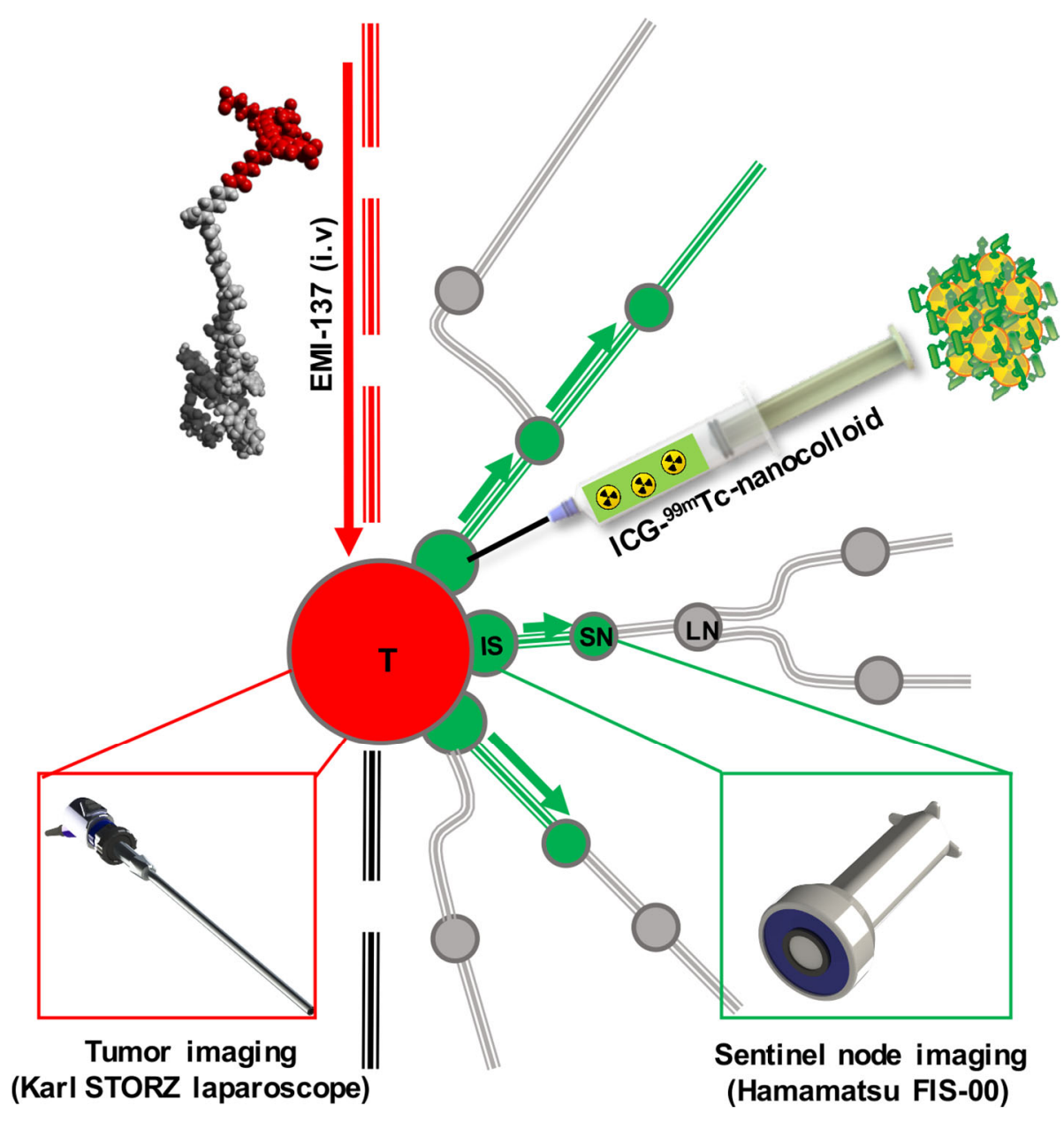

Figure 1. Logistics of hybrid and multicolour imaging in penile cancer patients. Right) Local administration of the

hybrid tracer ICG- ${ }^{99 m}$ Tc-nanocolloid (SN imaging; $\lambda_{\max e x}=800 \mathrm{~nm} ; \lambda_{\max e m}=820 \mathrm{~nm}$ ) is followed by SN identification based on planar lymphoscintigraphy and SPECT/CT at 15 minutes and $2 \mathrm{hrs}$. During the surgical procedure (4-24 hours post tracer administration), preoperative images serve as a personalized surgical roadmap for intraoperative radioand fluorescence-guided SN localization. ICG-based fluorescence guidance was achieved using the PDE-FIS from Hamamatsu Photonics (3). Left) Fluorescence-guided tumour visualization was achieved at 3 hours after intravenous administration of the Cy5-fluorescent C-Met targeting tracer EMI-137 $\left(\lambda_{\max }\right.$ ex $=640 \mathrm{~nm} ; \lambda_{\max }$ em $\left.=680 \mathrm{~nm}\right)$. Intraoperative imaging was achieved through the (first-in-human) use of a clinical grade Cy5-dedicated Laparoscope from STORZ Endoskope (11). 


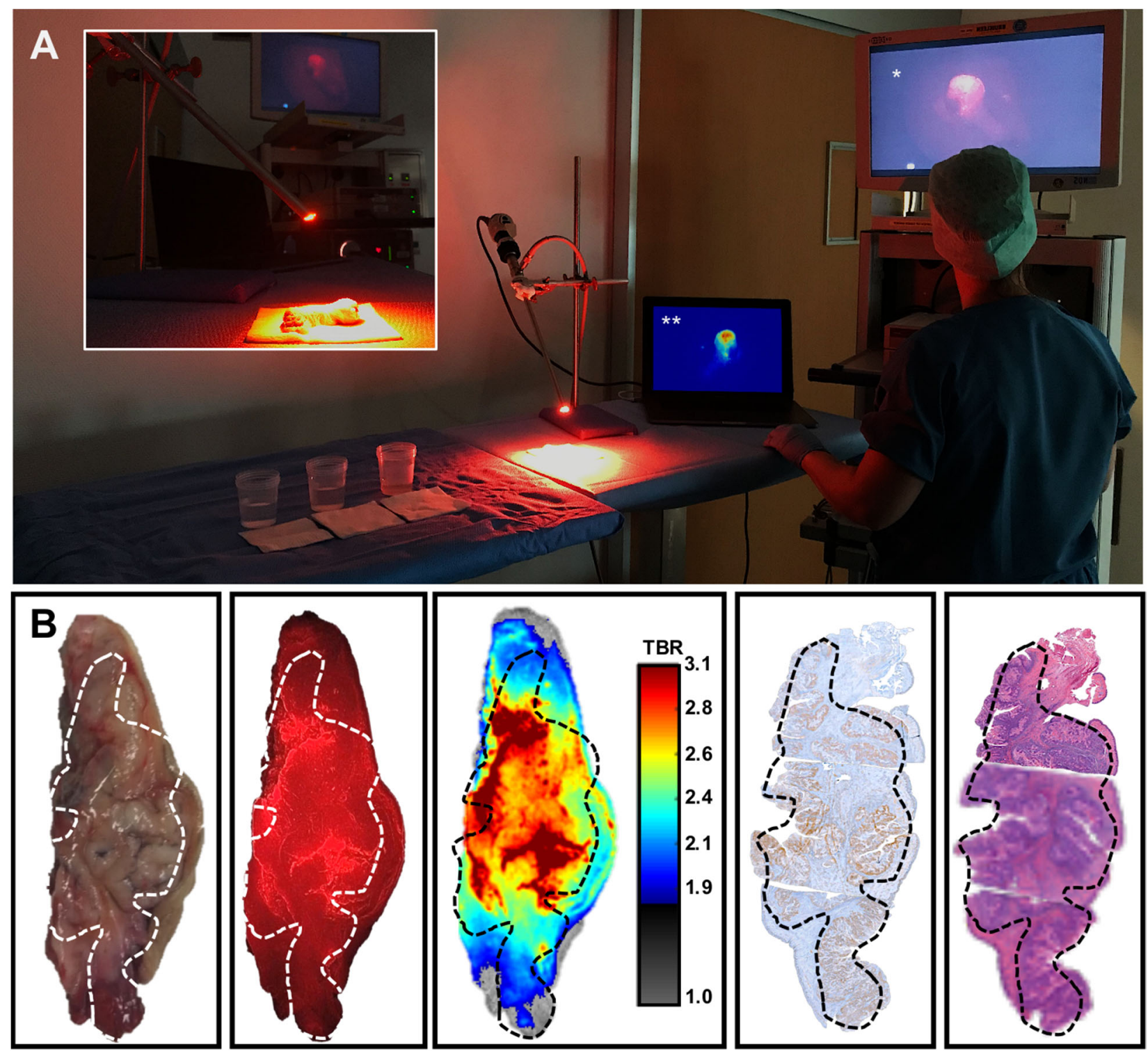

Figure 2. Ex vivo c-MET-targeted imaging in penile cancer. A) For ex vivo assessment of targeting feasibility, pSCC

tissue samples obtained after surgical tumour excision in penile cancer patients were incubated in a solution containing EMI-137 (five minutes; on the left, including two washing steps). Imaging was performed using the STORZ Cy5 laparoscope, where after the results were depicted onto two screens showing * the unprocessed and $* *$ realtime processed fluorescence image. Insert: detailed view of the illumination of the tissue sample. B) White-light image of excised tissue sample (left). Ex vivo Cy5-based fluorescence imaging of the incubated tissue (second from left; fluorescence in red) and real-time image processing of the fluorescence signal (centre; heatmap colour coding) correlated to c-MET immunohistochemistry (second from right) and H\&E staining (right) of the tissue samples. Tumour delineation (dashed) was based on H\&E. 

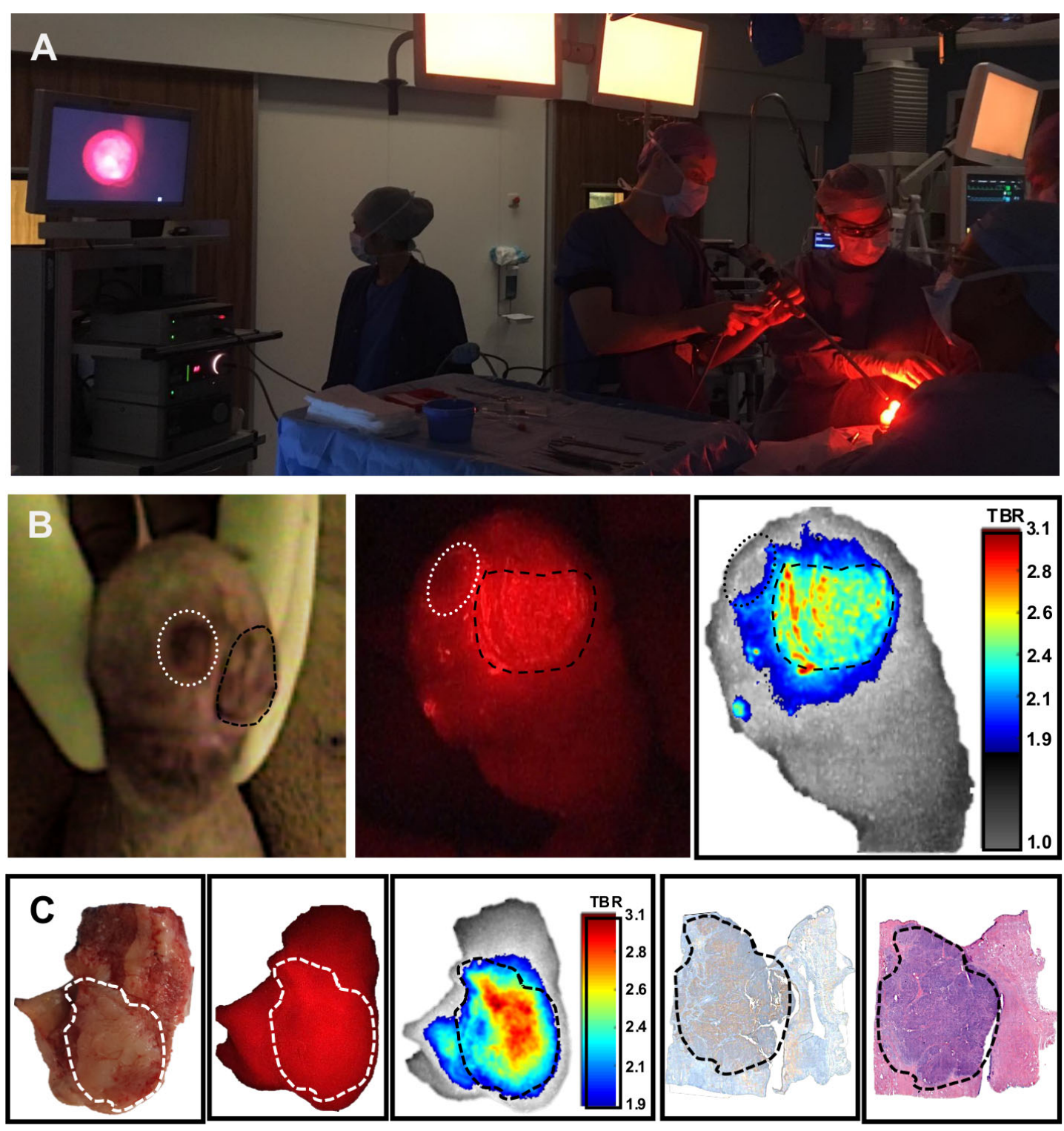

Figure 3. Tumour imaging. A) Overview of the surgical set-up within the operating room. B) Pre-incision imaging of the penis (left) showing both the urethral orifice (urinary meatus) and the tumour (encircled in black) with Cy5-based localization of the tumour (centre) and real-time colour-coding of the fluorescence signal (right, with colour coded scalebar representing the tumour-to-background ratio (TBR)). The dotted white line indicates an ulcus after biopsy with at pathological examination penile intraepithelial neoplasia. C) Ex vivo imaging of the excised tumour specimen (left) with Cy5-based fluorescence in red (second to left) and real-time image processing (centre) of the fluorescence signal (heatmap colour coding) correlated to c-MET immunohistochemistry (second to right) and H\&E staining (right) of the tissue samples. Tumour delineation (dashed) was based on H\&E. 

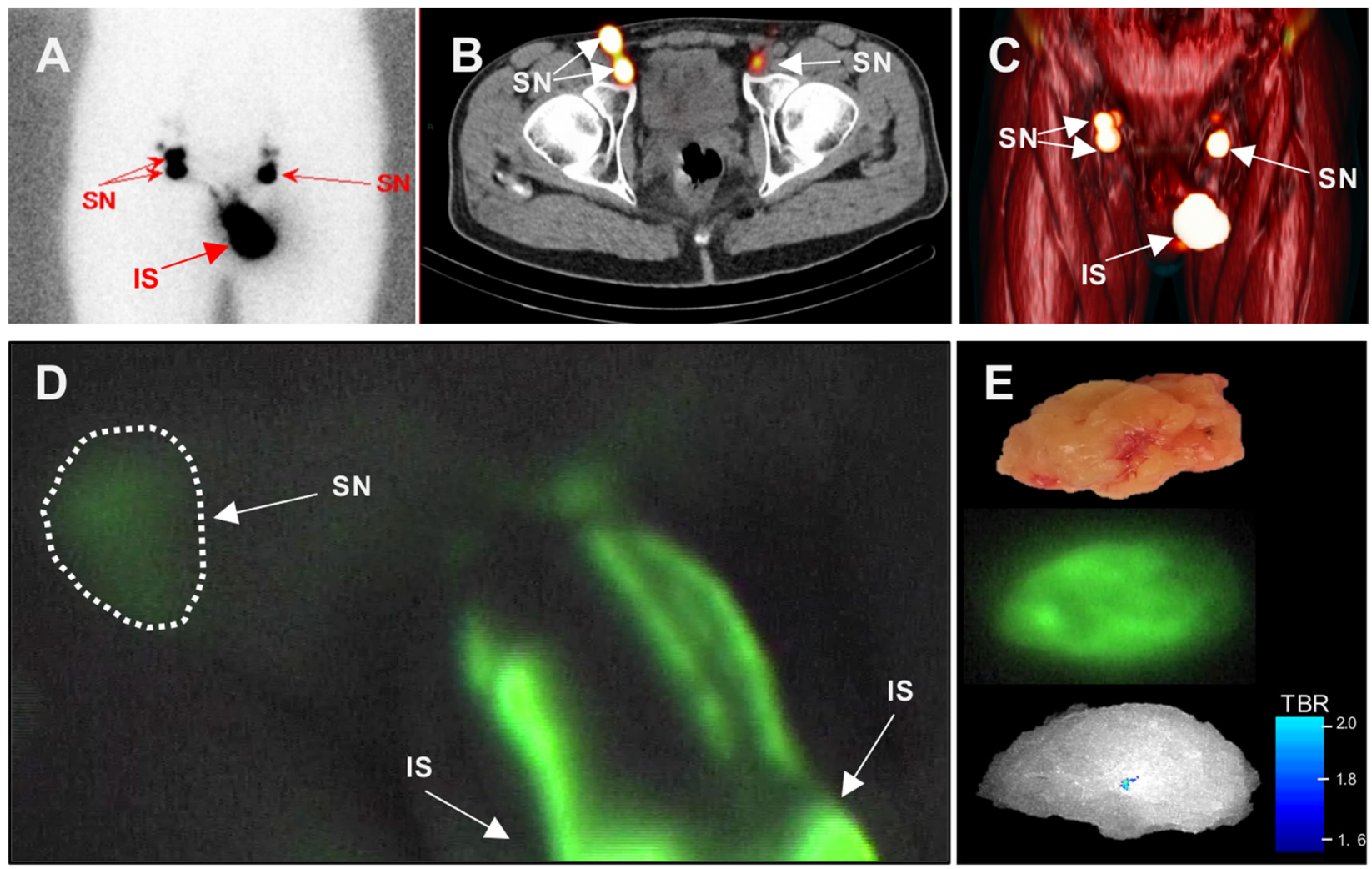

Figure 4. Sentinel node imaging. A) Standard lymphography after local administration of ICG- ${ }^{99 m}$ TC-nanocolloid specifying the SNs. B) Accompanying SPECT/CT image and C) 3D representation of the SPECT/CT results placing the SNs in their anatomical context. IS = injection site, $\mathrm{SN}=$ sentinel node. D) Pre-incision ICG-based fluorescence image showing the injection site of ICG-99mTc-nanocolloid with drainage through the lymphatics towards the SN (encircled). C) Post-excision imaging of the SN (white light, top image) with ICG in green (centre) and infra-red imaging after image processing (bottom image, including colour-coded scalebar representing the tumour-to-background ratio (TBR)). 
Graphical Abstract

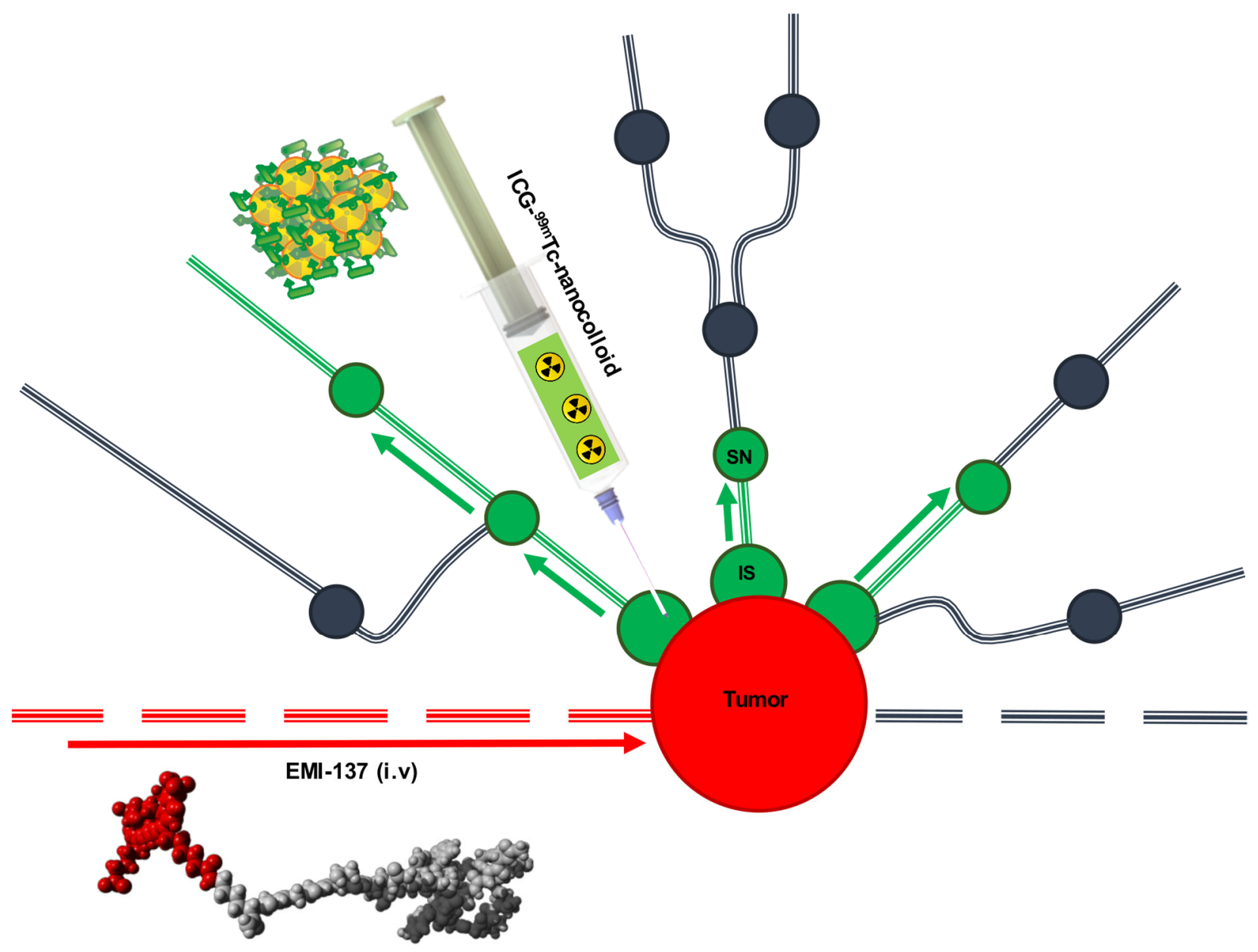




\begin{tabular}{|c|c|c|c|c|c|c|c|c|c|c|c|c|c|c|c|c|c|c|}
\hline & $\begin{array}{l}\text { Time } \\
\text { injection to } \\
\text { surgery } \\
\text { (min) }\end{array}$ & cT & $\mathrm{cN}$ & cM & Surgery & pT & $\mathrm{pN}$ & pGr & PeIN & LVI & PNI & $\mathbf{R}$ & $\begin{array}{l}\text { Diameter } \\
(\mathrm{mm})\end{array}$ & $\begin{array}{l}\text { Infiltration } \\
\text { depth } \\
\text { (mm) }\end{array}$ & p16 & $\begin{array}{c}\text { c-MET } \\
\text { expression } \\
(\%)\end{array}$ & $\begin{array}{c}\text { c- } \\
\text { MET } \\
\text { score }\end{array}$ & TBR \\
\hline Ex vivo & & 2 & 1 & 0 & $\begin{array}{c}\text { Partial } \\
\text { penectomy }\end{array}$ & 3 & 1 & 2 & no & yes & yes & yes & 28 & 24 & pos & 70 & 0,7 & 3,2 \\
\hline Ex vivo & & 2 & 0 & $x$ & Re-circumcision & $1 a$ & 0 & 2 & yes & no & no & yes & 5 & 2 & neg & 95 & 0,85 & 2 \\
\hline Ex vivo & & 2 & 0 & $x$ & $\begin{array}{c}\text { Partial } \\
\text { penectomy }\end{array}$ & $1 a$ & 0 & 1 & yes & no & no & yes & 9 & 2 & neg & 90 & 1,35 & 2,8 \\
\hline Ex vivo & & 2 & 0 & $x$ & $\begin{array}{l}\text { Local excision } \\
\text { with circumcision }\end{array}$ & $1 a$ & 0 & 2 & yes & no & no & yes & 25 & 3 & neg & 90 & 0,9 & 2,5 \\
\hline Ex vivo & & 2 & 2 & 0 & $\begin{array}{c}\text { Local excision } \\
\text { with circumcision }\end{array}$ & $1 a$ & $x$ & 2 & no & no & - & yes & 48 & 17 & neg & 70 & 1,5 & 2,2 \\
\hline Ex vivo & & 3 & 0 & $x$ & $\begin{array}{c}\text { Subtotal } \\
\text { penectomy }\end{array}$ & 3 & $x$ & 3 & no & yes & yes & no & 52 & - & neg & 90 & 0,9 & 2,6 \\
\hline Ex vivo & & 2 & 0 & $x$ & $\begin{array}{c}\text { Local excision } \\
\text { with circumcision }\end{array}$ & $1 a$ & 0 & 2 & no & no & no & yes & 43 & 5 & pos & 80 & 1,1 & 3,4 \\
\hline Ex vivo & & 3 & 3 & 0 & $\begin{array}{c}\text { Partial } \\
\text { penectomy }\end{array}$ & 3 & 3 & 2 & no & yes & - & yes & 33 & 24 & pos & 100 & 2,3 & 2,9 \\
\hline Ex vivo & & 2 & 0 & $x$ & $\begin{array}{c}\text { Partial } \\
\text { penectomy }\end{array}$ & 3 & 0 & 3 & yes & yes & yes & yes & 21 & 14 & pos & 95 & 1,65 & 2,3 \\
\hline Ex vivo & & 2 & 0 & $x$ & $\begin{array}{c}\text { Partial } \\
\text { penectomy }\end{array}$ & 2 & 0 & 2 & no & yes & yes & yes & 55 & - & neg & 95 & 1,2 & 2,7 \\
\hline In vivo & 263 & 1 & 0 & $x$ & Local excision & $1 a$ & $x$ & 1 & yes & no & - & yes & 18 & 0,5 & - & 80 & 1,1 & 2,1 \\
\hline In vivo & 236 & 2 & 3 & 1 & $\begin{array}{c}\text { Partial } \\
\text { penectomy }\end{array}$ & 3 & $x$ & 3 & yes & yes & no & yes & 21 & - & pos & 100 & 1,75 & 4,2 \\
\hline In vivo & 245 & 2 & 0 & $x$ & $\begin{array}{l}\text { Local excision } \\
\text { and circumcision }\end{array}$ & $1 a$ & 0 & 2 & no & no & no & yes & 18 & 4,5 & neg & 30 & 0,4 & 2,1 \\
\hline In vivo & 202 & 2 & 0 & $x$ & $\begin{array}{l}\text { Local excision } \\
\text { and circumcision }\end{array}$ & $1 a$ & 0 & 2 & no & no & no & yes & 12 & 2 & pos & 100 & 2,3 & 2 \\
\hline In vivo & 183 & 1 & 0 & $x$ & $\begin{array}{c}\text { Partial } \\
\text { glansectomy }\end{array}$ & $1 a$ & 0 & 2 & no & no & yes & yes & 13 & 6 & neg & 90 & 1,55 & 3,3 \\
\hline
\end{tabular}


Table 1: Patient characteristics. All 15 ex vivo and in vivo patients and their particular tumour characteristics are presented here. cT = clinical T-stage, $\mathrm{cN}=$ clinical $\mathrm{N}$-stage, $\mathrm{cM}=$ clinical $\mathrm{M}$-stage, $\mathrm{pT}=$ pathological $\mathrm{T}$-stage, $\mathrm{pN}=$ pathological $\mathrm{N}$-stage, $\mathrm{pGr}=$ pathological differentiation grade, PeIN = penile intraepithelial neoplasia, $\mathrm{LVI}=$ lymphovascular invasion, $\mathrm{PNI}=$ perineural invasion, $\mathrm{R}=$ radicality TBR $=$ tumour to background ratio, $x=$ not staged,$-($ a dash $)=$ not reported, pos $=$ positive, neg $=$ negative. 